\title{
DENSE MOLECULAR GAS AND THE ROLE OF STAR FORMATION IN THE HOST GALAXIES OF QUASI-STELLAR OBJECTS
}

\author{
A. S. Evans, ${ }^{1}$ P. M. Solomon, ${ }^{1}$ L. J. Tacconi, ${ }^{2}$ T. Vavilkin, ${ }^{1}$ and D. Downes ${ }^{3}$ \\ Received 2006 July 1; accepted 2006 August 14
}

\begin{abstract}
New millimeter-wave $\mathrm{CO}$ and $\mathrm{HCN}$ observations of the host galaxies of infrared-excess Palomar-Green (PG) quasistellar objects (QSOs) previously detected in $\mathrm{CO}$ are presented. These observations are designed to assess the validity of using the infrared luminosity to estimate star formation rates of luminous active galactic nuclei (AGNs) by determining the relative significance of dust heating by young, massive stars and AGNs in QSO hosts and IRAS galaxies with warm, AGN-like infrared colors. The analysis of these data is based, in part, on evidence that HCN traces highdensity $\left(>10^{4} \mathrm{~cm}^{-3}\right)$ molecular gas, and that the starburst-to-HCN luminosity ratio, $L_{\mathrm{SB}} / L_{\mathrm{HCN}}^{\prime}$, of IRAS-detected galaxies is constant. The new $\mathrm{CO}$ data provide a confirmation of prior claims that PG QSO hosts have high infrared-toCO luminosity ratios, $L_{\mathrm{IR}} / L_{\mathrm{CO}}^{\prime}$, relative to IRAS galaxies of comparable $L_{\mathrm{IR}}$. Such high $L_{\mathrm{IR}} / L_{\mathrm{CO}}^{\prime}$ ratios may be due to significant heating of dust by the QSO or to an increased star formation efficiency in QSO hosts relative to the bulk of the luminous IRAS galaxy population. The $\mathrm{HCN}$ data show a similar trend, with the PG QSO host I Zw 1 and most of the warm IRAS galaxies having high $L_{\mathrm{IR}} / L_{\mathrm{HCN}}^{\prime}(>1600)$ relative to the cool IRAS galaxy population, for which the median $\left\langle L_{\mathrm{IR}} / L_{\mathrm{HCN}}^{\prime}\right\rangle_{\text {cool }} \sim 890_{-470}^{+440}$. If the assumption is made that the infrared emission from cool IRAS galaxies is reprocessed light from embedded star-forming regions, then high values of $L_{\mathrm{IR}} / L_{\mathrm{HCN}}^{\prime}$ are likely the result of dust heating by the AGNs. Further, if the median ratio of $L_{\mathrm{HCN}}^{\prime} / L_{\mathrm{CO}}^{\prime} \sim 0.06$ observed for Seyfert galaxies and I Zw 1 is applied to the PG QSOs not detected in $\mathrm{HCN}$, then the derived $L_{\mathrm{IR}} / L_{\mathrm{HCN}}^{\prime}$ values correspond to a stellar contribution to the production of $L_{\mathrm{IR}}$ of $\sim 7 \%-39 \%$, and star formation rates of $\sim 2-37 M_{\odot} \mathrm{yr}^{-1}$ are derived for the QSO hosts. The corresponding values for the warm galaxies are $\sim 10 \%-100 \%$ and $\sim 3-220 M_{\odot} \mathrm{yr}^{-1}$. Alternatively, if the far-infrared is adopted as the star formation component of the total infrared in cool galaxies, i.e., $\left\langle L_{\mathrm{FIR}} / L_{\mathrm{HCN}}^{\prime}\right\rangle_{\mathrm{cool}} \sim L_{\mathrm{SB}} / L_{\mathrm{HCN}}^{\prime}$, the stellar contributions in QSO hosts and warm galaxies to their $L_{\mathrm{FIR}}$ are up to $35 \%$ and $10 \%$ higher, respectively, than the percentages derived for $L_{\mathrm{IR}}$. This raises the possibility that the $L_{\mathrm{FIR}}$ in several of the PG QSO hosts, including I Zw 1, could be due entirely to dust heated by young, massive stars. Finally, there is no evidence that the global HCN emission is enhanced relative to $\mathrm{CO}$ in galaxies hosting luminous AGNs.
\end{abstract}

Key words: galaxies: active — galaxies: interactions — galaxies: ISM — infrared: galaxies — ISM: molecules — quasars: general

\section{INTRODUCTION}

Over the last two decades, considerable progress has been made in investigating the possible evolutionary connection between ultraluminous infrared galaxy (ULIG, i.e., $L_{\mathrm{IR}}[8-1000 \mu \mathrm{m}] \geq$ $10^{12} L_{\odot}$ ) mergers and quasi-stellar objects (QSOs) first proposed by Sanders et al. (1988b, 1988c). These studies have transformed our view of both the environments in which QSOs are triggered and fed and the connection between star formation and active galactic nucleus (AGN) activity.

Approximately $70 \%$ of the optically selected Palomar-Green (PG) QSO sample have Infrared Astronomical Satellite (IRAS) detections consistent with thermal emission from dust (Sanders et al. 1989). In addition, approximately a dozen low-redshift QSOs have been detected in $\mathrm{CO}(1 \rightarrow 0)$ to date (Sanders et al. 1988a; Barvainis et al. 1989; Alloin et al. 1992; Evans et al. 2001; Scoville et al. 2003), indicating substantial quantities of molecular gas out of which to fuel star formation and possibly AGN activity.

The detection of $\mathrm{CO}(1 \rightarrow 0)$ emission does not, in itself, imply that active star formation is occurring. Furthermore, in galaxies

\footnotetext{
${ }^{1}$ Department of Physics and Astronomy, State University of New York at Stony Brook, Stony Brook, NY 11794-3800; aevans@mail.astro.sunysb.edu, psolomon@sbastk.ess.sunysb.edu, tvavilk@vulcan.ess.sunysb.edu.

2 Max-Planck-Institut fur Extraterrestrische Physik, Giessenbachstrasse, Garching D-85748, Germany; linda@mpe.mpg.de.

${ }^{3}$ Institut de Radio Astronomie Millimetrique, Domaine Universitaire, F-38406 St. Martin d'Heres, France; downes@iram.fr.
}

known to harbor AGNs, it is unclear what fraction of the infrared emission is reprocessed starlight or AGN light; thus, using $L_{\mathrm{IR}}$ to estimate star formation rates may not be justified. The present paper addresses these issues by reporting the results of an $\mathrm{HCN}(1 \rightarrow 0)$ survey of QSOs previously detected in CO. HCN has a higher dipole moment and larger Einstein $A$-coefficient than $\mathrm{CO}$, and thus traces much denser gas $\left(\gtrsim 10^{4} \mathrm{~cm}^{-3}\right)$. In the Galaxy HCN emission is associated with molecular-cloud cores where active star formation is occurring, and it has been detected in a large number of infrared luminous galaxies (Gao \& Solomon 2004a). Gao \& Solomon (2004b) have shown that, for spiral and infrared luminous galaxies spanning several orders in $L_{\mathrm{IR}}$, the infrared-to$\mathrm{HCN}$ luminosity ratio, $L_{\mathrm{IR}} / L_{\mathrm{HCN}}^{\prime}$, is relatively constant and has smaller deviations than $L_{\mathrm{IR}} / L_{\mathrm{CO}}^{\prime}$ measured over the same $L_{\mathrm{IR}}$ range. Gao \& Solomon concluded that HCN is a much better tracer of the gas that ultimately gives rise to the young stellar population in these galaxies, and that the constancy of $L_{\mathrm{IR}} / L_{\mathrm{HCN}}^{\prime}$ is also proof that the $L_{\mathrm{IR}}$ is due entirely to dust heating by young, massive stars. With this as a working assumption, the data presented here are used to make a comparison between the $L_{\mathrm{IR}} / L_{\mathrm{HCN}}^{\prime}$ of the QSO host sample and the IRAS galaxy sample. Similarities between the $L_{\mathrm{IR}} / L_{\mathrm{HCN}}^{\prime}$ ratios of the bulk of the IRAS galaxy population and those of QSO and IRAS galaxies with warm, AGN-like infrared colors are expected if the $L_{\mathrm{IR}}$ of QSO hosts and warm galaxies is due to dust heating by young, massive stars.

This paper is divided into six sections. Section 2 is a summary of the sample selection. The observing procedure and data reduction 
TABLE 1

SOURCE LIST

\begin{tabular}{|c|c|c|c|c|c|c|c|}
\hline Source & R.A. (J2000.0) & Decl. (J2000.0) & $\begin{array}{c}D_{L}^{\mathrm{a}} \\
(\mathrm{Mpc})\end{array}$ & $z_{\mathrm{CO}}$ & $\begin{array}{c}L_{\mathrm{IR}}^{\mathrm{a}} \\
\left(\times 10^{11} L_{\odot}\right)\end{array}$ & $\begin{array}{c}L_{\mathrm{FIR}}{ }^{\mathrm{a}} \\
\left(\times 10^{11} L_{\odot}\right)\end{array}$ & $\begin{array}{c}\mathrm{SFR}^{\mathrm{b}} \\
\left(M_{\odot} \mathrm{yr}^{-1}\right)\end{array}$ \\
\hline PG $0050+124=$ I Zw $1 \ldots \ldots \ldots \ldots \ldots \ldots$ & 005334.94 & +124136.20 & 250 & 0.061 & 7.2 & 2.8 & 95 \\
\hline PG $0157+001=$ Mrk $1014 \ldots \ldots \ldots \ldots \ldots$ & 015950.21 & +002340.62 & 680 & 0.163 & 32 & 21 & 420 \\
\hline 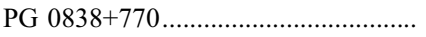 & 084445.36 & +765309.20 & 540 & 0.131 & 2.9 & 1.4 & 40 \\
\hline 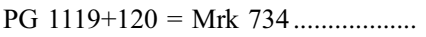 & 112147.15 & +114419.00 & 200 & 0.050 & 1.1 & 0.44 & 15 \\
\hline 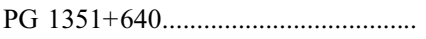 & 135315.83 & +634545.60 & 360 & 0.088 & 5.6 & 2.1 & 75 \\
\hline 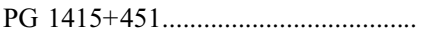 & 141700.84 & +445606.50 & 470 & 0.114 & 1.6 & 0.66 & 20 \\
\hline PG $1440+356=\operatorname{Mrk} 478 \ldots \ldots \ldots \ldots \ldots \ldots$ & 144207.48 & +352623.10 & 320 & 0.078 & 2.8 & 1.4 & 40 \\
\hline 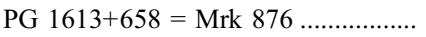 & 161357.21 & +654310.60 & 530 & 0.129 & 7.6 & 3.9 & 100 \\
\hline $\begin{array}{r}\text { PG } 2130+099=\text { UGC } 11763 \ldots \ldots \ldots \ldots \\
\text { Average } \ldots \ldots \ldots \ldots \ldots \ldots \ldots \ldots \ldots \ldots \ldots \ldots\end{array}$ & 213227.77 & +100819.50 & 250 & 0.063 & 2.1 & 0.62 & $\begin{array}{c}30 \\
90 \pm 125\end{array}$ \\
\hline 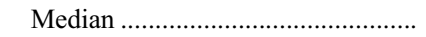 & & & & & & & 40 \\
\hline
\end{tabular}

Nоте.-Units of right ascension are hours, minutes, and seconds, and units of declination are degrees, arcminutes, and arcseconds.

${ }^{a}$ Calculated using the method in Sanders \& Mirabel (1996) and assuming $H_{0}=75 \mathrm{~km} \mathrm{~s}^{-1} \mathrm{Mpc}^{-1}$ and $q_{0}=0.5$. The $L_{\mathrm{IR}}$ of PG $1119+120$ and PG $2130+099$ are calculated using the flux densities published by Haas et al. (2003). For the remaining QSO host galaxies, the flux densities published by Sanders et al. (1989) are used.

${ }^{\mathrm{b}}$ Calculated by assuming that $L_{\mathrm{IR}}=L_{\mathrm{SB}}($ see $\S 5)$.

are presented in $\S 3$, followed by the results in $\S 4$. In $\S 5$ the infrared, $\mathrm{CO}$, and $\mathrm{HCN}$ data of the PG QSO hosts are compared with those of both cool and warm IRAS galaxies to assess the relative contribution of star formation to the infrared emission of luminous AGN hosts. Section 6 is a summary of the paper. Parts of this discussion have been presented elsewhere (Evans 2003, 2006a, 2006b). Throughout this paper we adopt an $H_{0}=$ $75 \mathrm{~km} \mathrm{~s}^{-1} \mathrm{Mpc}^{-1}$ and $q_{0}=0.5$ cosmology for consistency with Evans et al. (2001, 2005).

\section{SAMPLE}

The sample was selected from PG QSO hosts (Schmidt \& Green 1983) detected in $\mathrm{CO}(1 \rightarrow 0)$ to date (see Table 1). The sample is a subset of the QSO hosts imaged at optical and nearinfrared wavelengths by Surace et al. (2001) and defined to have infrared excesses (infrared-to-"big blue bump" luminosity ratio $\left.L_{\mathrm{IR}}[8-1000 \mu \mathrm{m}] / L_{\mathrm{bbb}}[0.1-1.0 \mu \mathrm{m}]>0.36\right)$ at least as high as those of ULIGs with warm, AGN-like infrared colors (i.e., $f_{25} \mu \mathrm{m} / f_{60 \mu \mathrm{m}} \geq$ 0.2 ; see Sanders et al. [1988c] for a discussion of the warm ULIG sample). Within the framework of the ULIG-QSO evolutionary model, infrared-excess PG QSO hosts represent the stage following the warm, AGN-like ULIG phase, and thus their hosts should still show signatures consistent with ultraluminous, gas-rich galaxy mergers (tidal tails, young star clusters, molecular gas, etc.). Given this, they are also the most likely QSO hosts to have the highest contribution of starlight to the production of their infrared luminosity. The reader is referred to Surace et al. (2001) for a more detailed discussion of the sample selection.

For the purposes of the study presented here, the QSOs listed in Table 1 have three primary advantages. First, the CO redshifts and line widths are known, thus making it straightforward to tune to the frequency of the HCN line and, in the absence of a strong HCN line, to measure upper limits. Second, IRAS and Infrared Space Observatory data have been published for these QSOs, making determinations of the infrared luminosity possible (Table 1). Third, because of the manner in which they are selected, they are directly comparable to the warm IRAS galaxy sample.

\section{OBSERVATIONS AND DATA REDUCTION}

Observations of the redshifted $\mathrm{HCN}$ emission were done at the IRAM $30 \mathrm{~m}$ telescope during five observing periods between 2003 May and 2003 December (see Table 2). The 3 and $2 \mathrm{~mm}$ receivers were used in combination with the filter-banks and an autocorrelator to provide bandwidths of 512 and $600 \mathrm{MHz}$, respectively. All QSO hosts with $\mathrm{HCN}(1 \rightarrow 0)$ emission redshifted to frequencies accessible with the $3 \mathrm{~mm}$ receivers were observed in $\mathrm{HCN}(1 \rightarrow 0)$; otherwise, $\mathrm{HCN}(2 \rightarrow 1)$ observations were obtained with the $2 \mathrm{~mm}$ receiver. During observations the pointing was monitored by observing standard continuum sources.

Three additional sets of observations were obtained. First, all of the QSOs were observed in $\mathrm{CO}(1 \rightarrow 0)$ during the 2003 December and 2006 May observing periods to verify CO redshifts and line widths. Second, observations of the $\mathrm{HCN}(1 \rightarrow 0)$ emission from Arp 220 and the $\mathrm{CO}(1 \rightarrow 0)$ emission from VII Zw 031 were made after every other QSO observation as a reality check of the $30 \mathrm{~m}$ telescope system performance. Finally, Arp 220 was observed in $\operatorname{HCN}(1 \rightarrow 0)$ and $\operatorname{HCN}(2 \rightarrow 1)$ to measure the ratio of two lines (see $\S 4$ ).

The data were reduced using the IRAM data reduction package CLASS. Scans were averaged together, and a linear baseline was subtracted from each spectrum outside of the velocity range

TABLE 2

Journal of Observations

\begin{tabular}{|c|c|c|c|c|}
\hline Source & Transition & Date & $\begin{array}{l}\text { Time } \\
\text { (hr) }\end{array}$ & $\begin{array}{l}T_{\text {sys }} \\
(\mathrm{K})\end{array}$ \\
\hline \multirow[t]{3}{*}{ PG $0050+124 \ldots \ldots \ldots$} & $\mathrm{CO}(1 \rightarrow 0)$ & $2003 \mathrm{Dec}$ & 40 & 161 \\
\hline & $\mathrm{HCN}(1 \rightarrow 0)$ & 2003 May & 527 & 102 \\
\hline & $\mathrm{HCN}(1 \rightarrow 0)$ & $2003 \mathrm{Sep}$ & 215 & 111 \\
\hline \multirow[t]{2}{*}{ PG $0157+001 \ldots \ldots .}$. & $\mathrm{CO}(1 \rightarrow 0)$ & $2003 \mathrm{Dec}$ & 50 & 111 \\
\hline & $\mathrm{HCN}(1 \rightarrow 0)$ & $2003 \mathrm{Dec}$ & 70 & 260 \\
\hline \multirow[t]{2}{*}{ PG $0838+770 \ldots \ldots .}$. & $\mathrm{CO}(1 \rightarrow 0)$ & 2003 Dec & 45 & 111 \\
\hline & $\mathrm{HCN}(2 \rightarrow 1)$ & 2003 May & 220 & 217 \\
\hline \multirow[t]{2}{*}{ PG $1119+120 \ldots \ldots .}$. & $\mathrm{CO}(1 \rightarrow 0)$ & 2003 Dec & 67 & 95 \\
\hline & $\mathrm{HCN}(1 \rightarrow 0)$ & 2003 Jun & 301 & 108 \\
\hline \multirow[t]{2}{*}{ PG $1351+640 \ldots \ldots \ldots$} & $\mathrm{CO}(1 \rightarrow 0)$ & 2006 May & 98 & 146 \\
\hline & $\mathrm{HCN}(1 \rightarrow 0)$ & 2003 Jun & 300 & 111 \\
\hline \multirow[t]{2}{*}{ PG $1415+451 \ldots \ldots \ldots$} & $\mathrm{CO}(1 \rightarrow 0)$ & 2003 Dec & 50 & 106 \\
\hline & $\mathrm{HCN}(1 \rightarrow 0)$ & 2003 Dec & 205 & 116 \\
\hline \multirow[t]{2}{*}{ PG $1440+356 \ldots \ldots .}$. & $\mathrm{CO}(1 \rightarrow 0)$ & $2003 \mathrm{Dec}$ & 80 & 126 \\
\hline & $\mathrm{HCN}(1 \rightarrow 0)$ & $2003 \mathrm{Dec}$ & 210 & 120 \\
\hline \multirow[t]{2}{*}{ PG $1613+658 \ldots \ldots \ldots$} & $\mathrm{CO}(1 \rightarrow 0)$ & 2003 Dec & 35 & 126 \\
\hline & $\mathrm{HCN}(2 \rightarrow 1)$ & $2003 \mathrm{Dec}$ & 125 & 235 \\
\hline PG $2130+099 \ldots \ldots .$. & $\mathrm{CO}(1 \rightarrow 0)$ & 2003 Dec & 140 & 157 \\
\hline
\end{tabular}


of the $\mathrm{CO}$ and $\mathrm{HCN}$ line emission, or, in the cases for which no $\mathrm{HCN}$ line was detected, outside the velocity range where the HCN line was expected to be. The amplitudes of each spectrum were converted to main-beam brightness temperatures, $T_{\mathrm{mb}}$, by multiplying the spectrum by the ratio of the forward-to-beam efficiencies of the telescope at the specific frequency of the observation and then smoothing each spectrum to $\sim 20 \mathrm{~km} \mathrm{~s}^{-1}$. Finally, line fluxes were measured by numerically integrating over the channels in the line profile, and the line widths were measured as full width at $50 \%$ of the peak flux. The resulting spectra are plotted in Figures 1-3.

\section{RESULTS}

\section{1. $\mathrm{CO}$}

Table 3 contains a summary of the CO emission-line properties of the sample of PG QSO hosts. The CO luminosities are calculated via

$$
\begin{aligned}
L_{\mathrm{CO}}^{\prime}= & 2.4 \times 10^{3}\left(\frac{S_{\mathrm{CO}} \Delta v}{1 \mathrm{Jy} \mathrm{km} \mathrm{s}^{-1}}\right)\left(\frac{D_{L}}{1 \mathrm{Mpc}}\right)^{2} \\
& \times(1+z)^{-1} \mathrm{~K} \mathrm{~km} \mathrm{~s}^{-1} \mathrm{pc}^{2}
\end{aligned}
$$

where $S_{\mathrm{CO}} \Delta v$ is the CO flux obtained by multiplying the line intensities in Table 3 by the conversion factor for an unresolved source (i.e., $S / T_{\mathrm{mb}}=4.95 \mathrm{~J} \mathrm{~K}^{-1}$ ) and $D_{L}$ is the luminosity distance. The molecular gas masses, $M_{\mathrm{H}_{2}}$, are calculated by adopting the conversion factor $\alpha=M\left(\mathrm{H}_{2}\right) / L_{\mathrm{CO}}^{\prime}=4 M_{\odot}\left(\mathrm{K} \mathrm{km} \mathrm{s}^{-1} \mathrm{pc}^{2}\right)^{-1}$ (see Evans et al. 2001 for a detailed discussion of $\alpha$ ).

There is considerable overlap between the QSOs listed in Table 3 and recent interferometric surveys with the Owens Valley Millimeter Array at the Owens Valley Radio Observatory (OVRO). Specifically, PG 0838+770, PG 1119+120, PG 1351+640, PG 1415+451, PG 1440+356, and PG 1613+658 were observed by Evans et al. (2001), and PG 2130+099 was observed as part of a volume-limited $(z<0.1)$ QSO CO survey by Scoville et al. (2003). In addition, previous single-dish measurements of CO emission from I Zw 1, Mrk 1014, PG 0838+770, and PG 1613+ 658 exist (Sanders et al. 1988a; Barvainis et al. 1989; Alloin et al. 1992).

The general shape and width of the $\mathrm{CO}$ lines are consistent with previously published CO spectra of these QSOs. For the QSOs with broad ( $>200 \mathrm{~km} \mathrm{~s}^{-1}$ ) CO emission lines, the CO velocity width measured at half the maximum intensity, $\Delta v_{\mathrm{FWHM}}$, agrees to within $20 \%$ of prior measurements. The agreement degrades to $40 \%$ for the narrow $\left(<100 \mathrm{~km} \mathrm{~s}^{-1}\right)$ CO line QSOs, the larger discrepancy resulting from the difficulty in accurately measuring $\Delta v_{\text {FWHM }}$ for narrow emission lines after rebinning the data. From these new measurements, an average $\mathrm{CO}$ emission line of $\Delta v_{\text {FWHM }} \sim 280 \pm 150 \mathrm{~km} \mathrm{~s}^{-1}$ is estimated, as compared to the value of $300 \pm 90 \mathrm{~km} \mathrm{~s}^{-1}$ measured for ULIGs (see Solomon et al. 1997; Evans et al. 2005).

With the exception of PG $1613+658$, the new measurements of main-beam CO line fluxes, $S_{\mathrm{CO}} \Delta v$, are on average $40 \%$ lower than the OVRO data presented in Evans et al. (2001). The new CO line flux measurement of PG 1613+658, which is the PG QSO with the highest signal-to-noise ratio $\mathrm{CO}$ detection in Evans et al. (2001), agrees to within 4\% with the OVRO data. Finally, the CO line flux of PG $2130+099$ agrees to within $10 \%$ of the measurement made by Scoville et al. (2003).

While previous single-dish $S_{\mathrm{CO}} \Delta v$ measurements of I Zw 1 and PG $1613+658$ are consistent to within $20 \%$ of the measurements presented here, single-dish measurements of PG $0838+$ 770 and Mrk 1014 are up to $300 \%$ higher than the present measurements. The discrepancies are undoubtedly a result of calibration errors and low signal-to-noise ratio data.

\section{2. $\mathrm{HCN}$}

Table 3 also summarizes the HCN emission-line properties of the QSO sample and Arp 220. Only the PG QSO host I Zw 1 was detected in $\mathrm{HCN}(1 \rightarrow 0)$; the shape and width of the HCN line are consistent with the $\mathrm{CO}(1 \rightarrow 0)$ profile. For the remaining PG QSO hosts, the HCN line intensities were measured by numerically integrating over the channels expected to contain $\mathrm{HCN}$ emission based on the $\mathrm{CO}(1 \rightarrow 0)$ observations. In addition, $3 \sigma$ upper limits to the HCN line intensities were derived via

$$
T_{\mathrm{HCN}} \Delta v<\frac{3 T_{\mathrm{rms}} \Delta v_{\mathrm{FWZI}}}{\sqrt{\Delta v_{\mathrm{FWZI}} / \Delta v_{\mathrm{res}}}} \mathrm{K} \mathrm{km} \mathrm{s}^{-1},
$$

where $\Delta v_{\text {FWZI }}$ is the full width at zero intensity velocity of the CO emission line of the QSO host and $T_{\text {rms }}$ is the rms main-beam temperature of the $\mathrm{HCN}$ spectral data for a velocity resolution of $\Delta v_{\text {res }}$. Line luminosities were calculated via

$$
\begin{aligned}
L_{\mathrm{HCN}(\mathrm{J} \rightarrow \mathrm{J}-1)}^{\prime}= & 4.145 \times 10^{3}\left(\frac{S_{\mathrm{HCN}} \Delta v}{1 \mathrm{Jy} \mathrm{km} \mathrm{s}^{-1}}\right)\left(\frac{D_{L}}{1 \mathrm{Mpc}}\right)^{2} \\
& \times J^{-2}(1+z)^{-1} \mathrm{~K} \mathrm{~km} \mathrm{~s}^{-1} \mathrm{pc}^{2} .
\end{aligned}
$$

The HCN $(1 \rightarrow 0)$ lines of two of the PG QSOs (PG $0838+770$ and $P G 1613+658)$ are redshifted to frequencies below the range accessible with the $3 \mathrm{~mm}$ receivers. In both cases, the $2 \mathrm{~mm}$ receivers were tuned to the frequencies corresponding to the redshifted $\mathrm{HCN}(2 \rightarrow 1)$ emission line. Both observations resulted in upper limits, which were converted to $\mathrm{HCN}(1 \rightarrow 0)$ upper limits by adopting the $L_{\mathrm{HCN}(1 \rightarrow 0)}^{\prime} / L_{\mathrm{HCN}(2 \rightarrow 1)}^{\prime}(=2.997)$ line ratio measured for Arp 220 (Fig. 3 ).

\section{DISCUSSION}

Approximately one-quarter of the PG QSOs at $z<0.3$ have been detected in $\mathrm{CO}(1 \rightarrow 0)$, and $\sim 50 \%-70 \%$ show evidence of spiral disks, merger-like tidal features, and/or luminous star clusters (e.g., Stockton \& MacKenty 1983; Surace et al. 2001 and references therein; Veilleux et al. 2006; Guyon et al. 2006). The presence of star-forming molecular gas and luminous star clusters, combined with infrared luminosities in excess of $10^{11} L_{\odot}$, are compelling indications that star formation is an important component of activity in these galaxies, and it raises the possibility that a significant fraction of their thermal infrared emission may be reprocessed stellar light.

Given such evidence, an obvious question to ask is: What are the star formation rates of the PG QSO hosts? As a naive first attempt, the assumption is made that the infrared luminosity of the host is due entirely to dust heating by the starburst population, and that $\tau \gg 1$ toward the star-forming regions. Doing so yields star formation rates of

$$
\begin{aligned}
\mathrm{SFR} & \sim 1.32 \times 10^{-10}\left(\frac{L_{\mathrm{IR}}}{1 L_{\odot}}\right)\left(\frac{\delta}{0.75}\right) M_{\odot} \mathrm{yr}^{-1} \\
& \sim 20-420 M_{\odot} \mathrm{yr}^{-1}
\end{aligned}
$$

with an average star formation rate of $\langle$ SFR $\rangle \sim 90 \pm 125 M_{\odot} \mathrm{yr}^{-1}$ and the median of $40 M_{\odot} \mathrm{yr}^{-1}$ (Table 1). Equation (4) is a modified version of equation (4) in Kennicutt (1998), where the factor $\delta(=0.75 \pm 0.07)$ is the average far-infrared-to-infrared luminosity ratio, $L_{\mathrm{FIR}}(40-500 \mu \mathrm{m}) / L_{\mathrm{IR}}(8-1000 \mu \mathrm{m})$, calculated for 

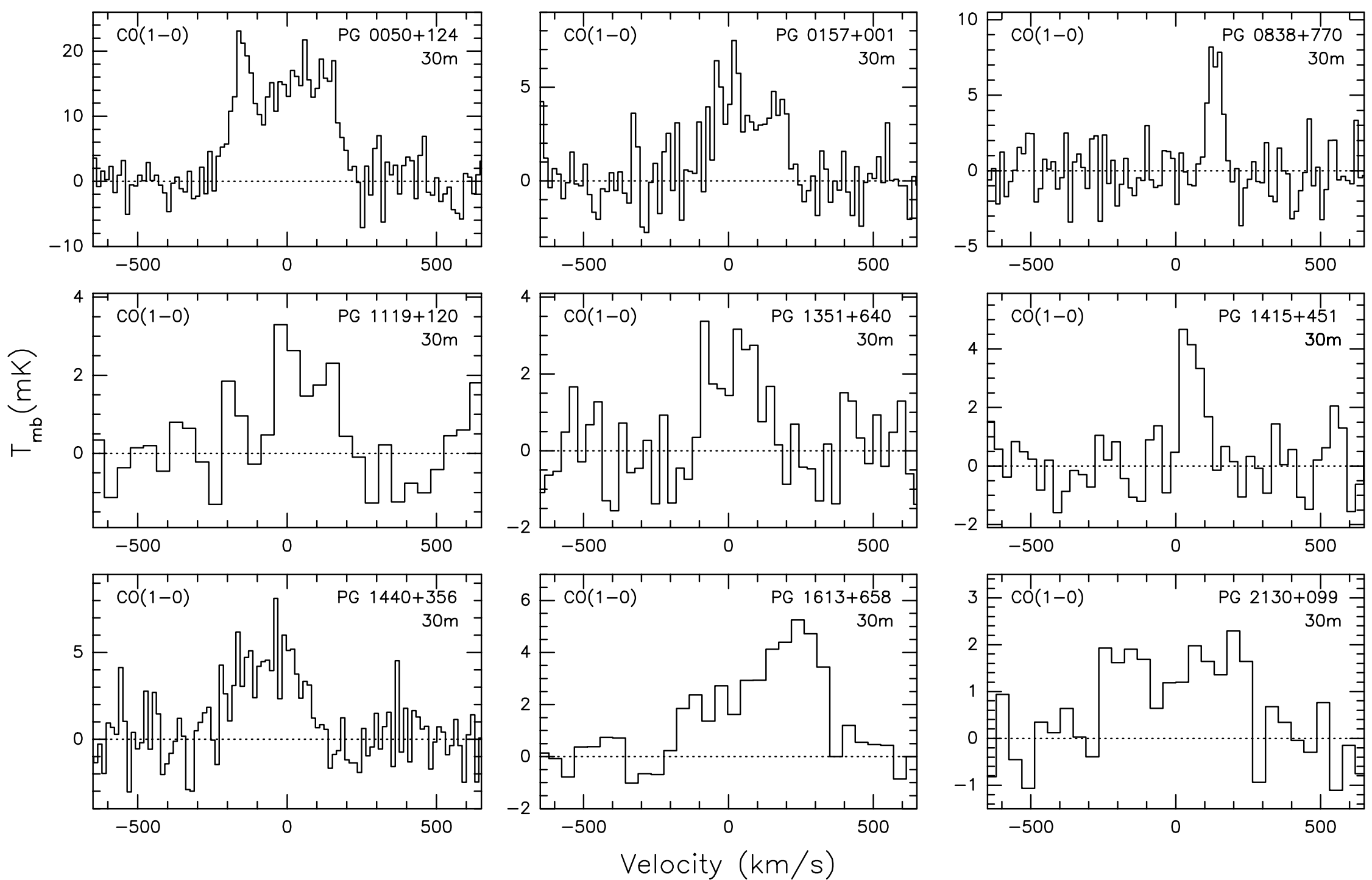

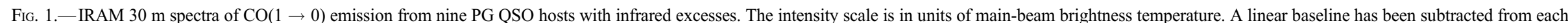
spectrum; the baseline subtraction is performed outside of the velocity range of the emission lines. The zero velocity corresponds to the redshifts listed in Table 1 . 

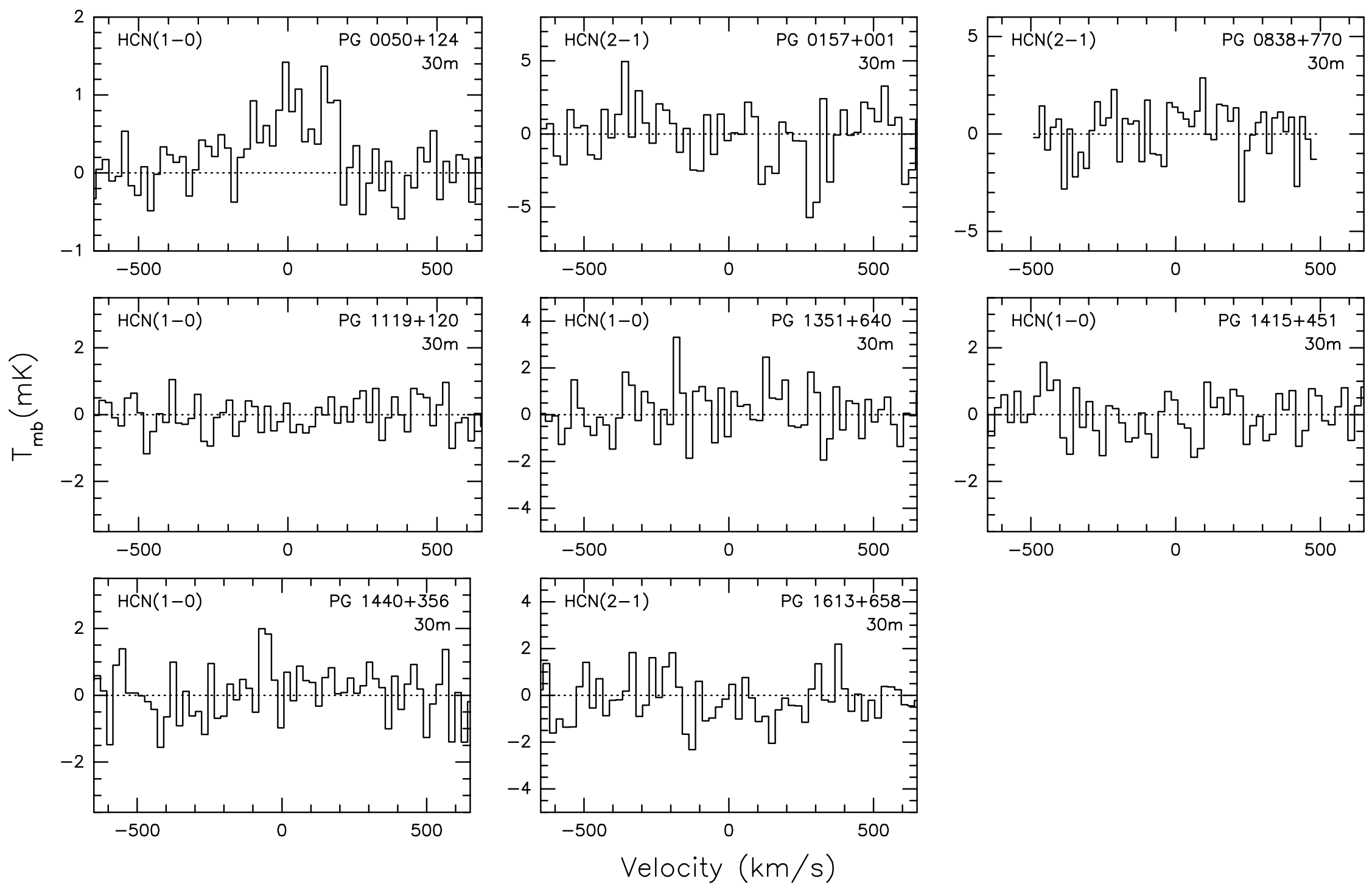

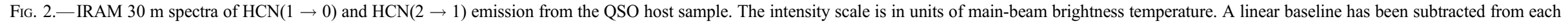
spectrum; the baseline subtraction is performed outside of the velocity range where the emission line is expected to be based on the position and velocity width of CO( $\rightarrow 0)$ emission from these QSO hosts. 


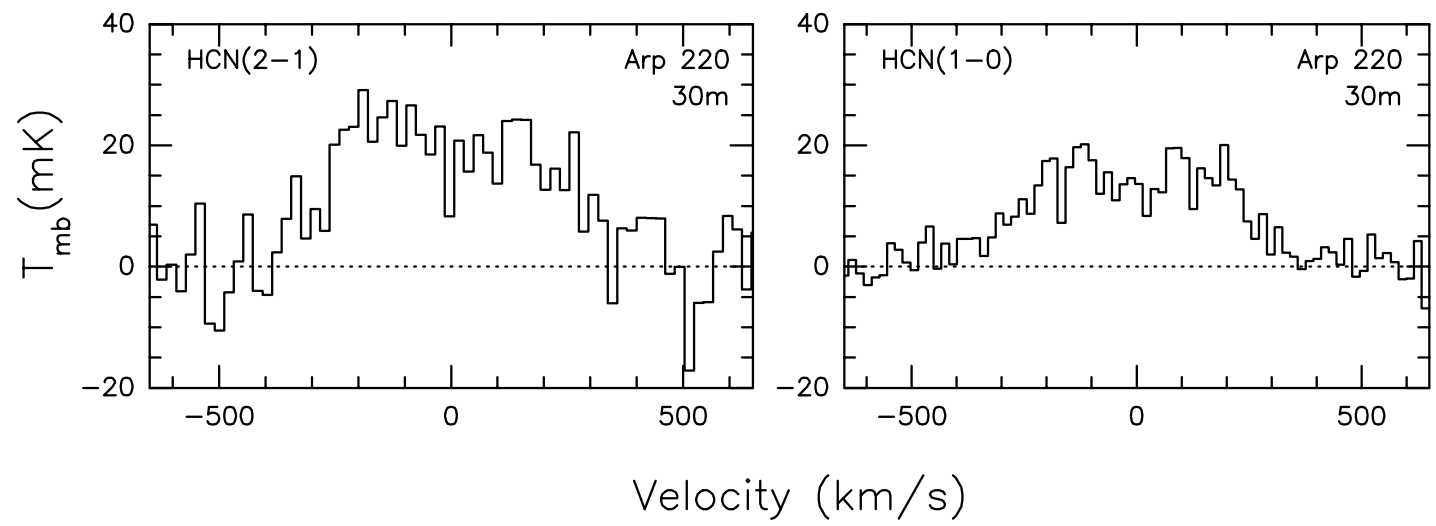

FIG. 3.- IRAM $30 \mathrm{~m}$ spectra of $\mathrm{HCN}(1 \rightarrow 0)$ and $\mathrm{HCN}(2 \rightarrow 1)$ emission from the ULIG Arp 220 . The intensity scale is in units of main-beam brightness temperature. A linear baseline has been subtracted from each spectrum; the baseline subtraction is performed outside of the velocity range of the emission lines. The zero velocity corresponds to a redshift of 0.0181 .

the IRAS galaxies in the Revised Bright Galaxy Sample $(\sim 600$ galaxies; Sanders et al. 2003). These star formation rates are likely upper limits; it is difficult to imagine a geometry in which light from the AGN does not intersect (and thus heat) a significant fraction of dust in the host galaxy. Indeed, models have been developed that can account for all of the infrared emission in QSO hosts by an AGN surrounded by a warped or clumpy torus (Sanders et al. 1989; see review in Haas et al. 2003; Elitzur et al. 2004).

\section{1. $L_{\mathrm{IR}} / L_{\mathrm{CO}}^{\prime}$ : Star Formation Efficiencies versus Dust Heating by the $A G N$}

To examine the importance of dust heating by young, massive stars to the production of $L_{\mathrm{IR}}$ in their hosts, consider first the $L_{\mathrm{CO}}^{\prime}$ and $L_{\mathrm{IR}}$ of QSO hosts relative to that of IRAS-detected galaxies
(Fig. 4; see also Evans et al. 2001, 2005). The new $L_{\mathrm{CO}}^{\prime}$ measurements of the infrared-excess QSO hosts (Table 3 ) are plotted in Figure $4{ }^{4}$ additional QSO data are compiled from Scoville et al. (2003). In order to provide a more meaningful comparison with the QSO hosts the IRAS galaxies have been separated into those with warm, Seyfert-like (i.e., $f_{25 \mu \mathrm{m}} / f_{60 \mu \mathrm{m}} \geq 0.20$ ) and cool $\left(f_{25 \mu \mathrm{m}} / f_{60 \mu \mathrm{m}}<0.20\right)$ infrared colors. There is significant evidence that most warm IRAS galaxies host AGNs, but there is not clear evidence that cool IRAS galaxies, as a class, have luminous

\footnotetext{
${ }^{4}$ Note that Evans et al. (2001) used $L_{\mathrm{IR}}(1-1000 \mu \mathrm{m})$ for QSO hosts instead of the commonly used $L_{\mathrm{IR}}(8-1000 \mu \mathrm{m})$. The $L_{\mathrm{IR}}(1-1000 \mu \mathrm{m})$ values in Evans et al. (2001) were calculated by numerically integrating the spectral energy distributions in Sanders et al. (1989). Here, we use $L_{\mathrm{IR}}(8-1000 \mu \mathrm{m})$, which leaves out the significant contribution of hot dust in the $1-8 \mu \mathrm{m}$ range but provides better consistency with the other data sets plotted.
}

TABLE 3

EMISSION-Line Properties

\begin{tabular}{|c|c|c|c|c|c|c|c|c|c|}
\hline Source & $z_{\mathrm{CO}}$ & Line & $\begin{array}{c}\Delta v_{\text {FWHM }} \\
\left(\mathrm{km} \mathrm{s}^{-1}\right)\end{array}$ & $\begin{array}{c}T_{\mathrm{mb}} \Delta v \\
\left(\mathrm{~K} \mathrm{~km} \mathrm{~s}^{-1}\right)\end{array}$ & $\begin{array}{c}S_{\text {line }} \Delta v \\
\left(\mathrm{Jy} \mathrm{km} \mathrm{s}^{-1}\right)\end{array}$ & $\begin{array}{c}L_{\text {line }}^{\prime}{ }^{\mathrm{a}, \mathrm{b}} \\
\left(\mathrm{K} \mathrm{km} \mathrm{s}^{-2} \mathrm{pc}^{2}\right)\end{array}$ & $\begin{array}{c}M\left(\mathrm{H}_{2}\right)^{\mathrm{c}} \\
\quad\left(M_{\odot}\right)\end{array}$ & $L_{\mathrm{IR}} / L_{\text {line }}^{\prime}{ }^{\mathrm{d}}$ & $\left.L_{\mathrm{IR}} / L_{\mathrm{HCN}(1 \rightarrow 0)}^{\prime}\right)^{\mathrm{d}, \mathrm{e}}$ \\
\hline \multirow[t]{2}{*}{ 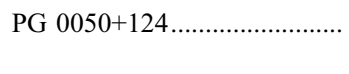 } & 0.061 & $\mathrm{CO}(1 \rightarrow 0)$ & 370 & $6.0 \pm 0.2$ & $30 \pm 1.1$ & $4.2 \times 10^{9}$ & $1.7 \times 10^{10}$ & 170 & $\ldots$ \\
\hline & 0.061 & $\mathrm{HCN}(1 \rightarrow 0)$ & 290 & $0.27 \pm 0.03$ & $1.3 \pm 0.1$ & $3.2 \times 10^{8}$ & & 2260 & $\ldots$ \\
\hline \multirow[t]{2}{*}{ PG $0157+001 \ldots \ldots \ldots \ldots \ldots \ldots \ldots \ldots$} & 0.163 & $\mathrm{CO}(1 \rightarrow 0)$ & 270 & $1.1 \pm 0.1$ & $5.5 \pm 0.5$ & $5.2 \times 10^{9}$ & $2.1 \times 10^{10}$ & 610 & $\ldots$ \\
\hline & $\ldots$ & $\mathrm{HCN}(1 \rightarrow 0)$ & $\ldots$ & $-0.12 \pm 0.16$ & $-0.60 \pm 0.80$ & $<9.6 \times 10^{8}$ & $\ldots$ & $>3300$ & $>1100$ \\
\hline \multirow[t]{2}{*}{ PG $0838+770 \ldots \ldots \ldots \ldots \ldots \ldots \ldots$} & 0.132 & $\mathrm{CO}(1 \rightarrow 0)$ & 60 & $0.50 \pm 0.07$ & $2.5 \pm 0.4$ & $1.5 \times 10^{9}$ & $6.1 \times 10^{9}$ & 190 & $\ldots$ \\
\hline & & $\mathrm{HCN}(2 \rightarrow 1)$ & $\ldots$ & $0.12 \pm 0.06$ & $0.60 \pm 0.31$ & $<1.8 \times 10^{8}$ & & $>1590$ & $>530$ \\
\hline \multirow[t]{2}{*}{ PG 1119+120 } & 0.050 & $\mathrm{CO}(1 \rightarrow 0)$ & 220 & $0.54 \pm 0.09$ & $2.7 \pm 0.4$ & $2.5 \times 10^{8}$ & $1.0 \times 10^{9}$ & 450 & $\ldots$ \\
\hline & & $\mathrm{HCN}(1 \rightarrow 0)$ & 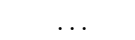 & $-0.01 \pm 0.04$ & $-0.05 \pm 0.19$ & $<9.2 \times 10^{7}$ & & $>1230$ & . . \\
\hline \multirow[t]{2}{*}{ 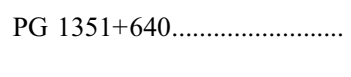 } & 0.088 & $\mathrm{CO}(1 \rightarrow 0)$ & 260 & $0.54 \pm 0.09$ & $2.7 \pm 0.5$ & $7.7 \times 10^{8}$ & $3.1 \times 10^{9}$ & 730 & $\ldots$ \\
\hline & & $\mathrm{HCN}(1 \rightarrow 0)$ & & $0.09 \pm 0.08$ & $0.46 \pm 0.40$ & $<5.5 \times 10^{8}$ & & $>1000$ & $\ldots$ \\
\hline \multirow[t]{2}{*}{ 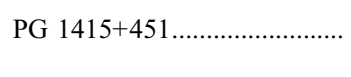 } & 0.114 & $\mathrm{CO}(1 \rightarrow 0)$ & 90 & $0.42 \pm 0.06$ & $2.1 \pm 0.3$ & $9.8 \times 10^{8}$ & $3.9 \times 10^{9}$ & 160 & $\ldots$ \\
\hline & & $\mathrm{HCN}(1 \rightarrow 0)$ & $\ldots$ & $-0.02 \pm 0.04$ & $-0.12 \pm 0.20$ & $<3.8 \times 10^{8}$ & $\ldots$ & $>420$ & $\ldots$ \\
\hline \multirow[t]{2}{*}{ 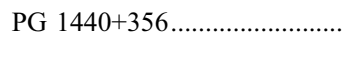 } & 0.078 & $\mathrm{CO}(1 \rightarrow 0)$ & 310 & $1.3 \pm 0.1$ & $6.6 \pm 0.6$ & $1.5 \times 10^{9}$ & $6.0 \times 10^{9}$ & 190 & $\ldots$ \\
\hline & & $\mathrm{HCN}(1 \rightarrow 0)$ & $\ldots$ & $0.09 \pm 0.08$ & $0.43 \pm 0.40$ & $<3.9 \times 10^{8}$ & & $>720$ & . \\
\hline \multirow[t]{2}{*}{ PG $1613+658 \ldots \ldots \ldots \ldots \ldots \ldots \ldots \ldots$} & 0.129 & $\mathrm{CO}(1 \rightarrow 0)$ & 400 & $1.6 \pm 0.1$ & $8.0 \pm 0.6$ & $4.8 \times 10^{9}$ & $1.9 \times 10^{10}$ & 160 & $\ldots$ \\
\hline & & $\mathrm{HCN}(2 \rightarrow 1)$ & $\ldots$ & $-0.17 \pm 0.13$ & $-0.83 \pm 0.63$ & $<4.1 \times 10^{8}$ & $\ldots$ & $>1850$ & $>620$ \\
\hline PG 2130+099 ……................. & 0.063 & $\mathrm{CO}(1 \rightarrow 0)$ & 530 & $0.78 \pm 0.1$ & $3.9 \pm 0.5$ & $5.6 \times 10^{8}$ & $2.2 \times 10^{9}$ & 380 & $\ldots$ \\
\hline \multirow[t]{2}{*}{ Arp $220 \ldots \ldots \ldots$} & 0.0181 & $\mathrm{HCN}(1 \rightarrow 0)$ & 550 & $9.0 \pm 0.8$ & $45 \pm 3$ & $9.6 \times 10^{8}$ & $\ldots$ & 1450 & $\ldots$ \\
\hline & (1) & $\mathrm{HCN}(2 \rightarrow 1)$ & 550 & $12 \pm 1.4$ & $60 \pm 7$ & $3.2 \times 10^{8}$ & $\ldots$ & $\ldots$ & $\ldots$ \\
\hline PG QSO average $(\mathrm{CO}) \ldots . .$. & & & $260 \pm 150$ & & & & & & \\
\hline
\end{tabular}

a $3 \sigma$ upper limit

b Calculated assuming $H_{0}=75 \mathrm{~km} \mathrm{~s}^{-1} \mathrm{Mpc}^{-1}$ and $q_{0}=0.5$.

${ }^{c}$ Calculated assuming $\alpha=4 M_{\odot}\left(\mathrm{K} \mathrm{km} \mathrm{s}^{-1} \mathrm{pc}^{2}\right)^{-1}$.

${ }^{\mathrm{d}}$ In units of $L_{\odot}\left(\mathrm{K} \mathrm{km} \mathrm{s}^{-1} \mathrm{pc}^{2}\right)^{-1}$.

e $L_{\mathrm{HCN}(2 \rightarrow 1)}^{\prime} / L_{\mathrm{HCN}(1 \rightarrow 0)}^{\prime}$ conversion is achieved by adopting the $L_{\mathrm{HCN}(1 \rightarrow 0)}^{\prime} / L_{\mathrm{HCN}(2 \rightarrow 1)}^{\prime}(=2.997)$ of Arp 220. See $\S 4.2$. 


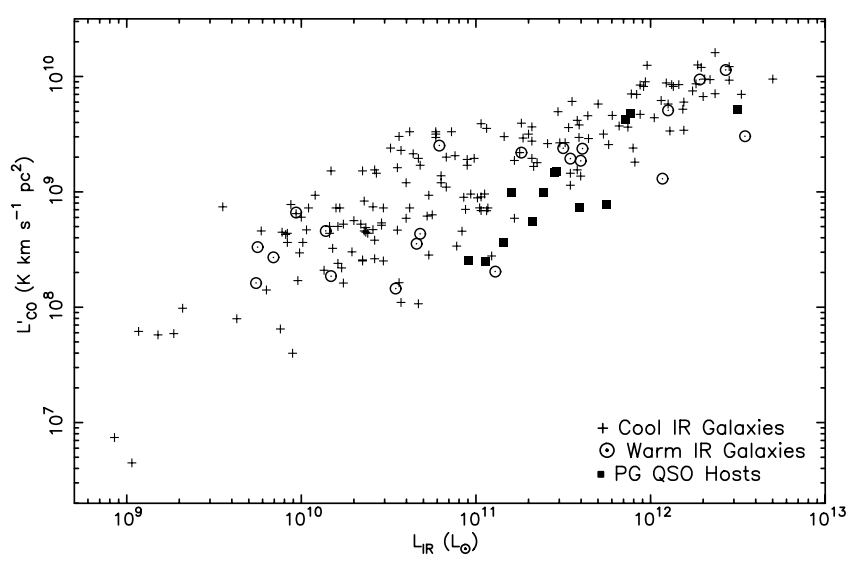

FIG. 4. $-L_{\mathrm{CO}}^{\prime}$ vs. $L_{\mathrm{IR}}$ of the PG QSO hosts and a sample of IRAS-detected galaxies. Infrared galaxy data come from Mazzarella et al. (1993) and Solomon et al. (1997), and PG QSO host data come from this paper and Scoville et al. (2003).

AGNs. Given this, the cool galaxies are treated as galaxies powered by starbursts.

The new CO data show results similar to what is shown in Evans et al. (2001, 2005); the QSO hosts have low $L_{\mathrm{CO}}^{\prime}$ for their $L_{\mathrm{IR}}$ relative to the bulk of cool IRAS galaxies. In other words, QSO hosts have high $L_{\mathrm{IR}} / L_{\mathrm{CO}}^{\prime}$ relative to cool IRAS galaxies with comparable $L_{\mathrm{IR}}$. The warm galaxies with $L_{\mathrm{IR}}>10^{10.0} L_{\odot}$ show a similar trend in $L_{\mathrm{IR}} / L_{\mathrm{CO}}^{\prime}$ relative to the cool IRAS sample, although not as dramatically as the QSO host population.

To interpret the data plotted in Figure 4, consider

$$
\frac{L_{\mathrm{IR}}}{L_{\mathrm{CO}}^{\prime}} \sim \frac{L_{\mathrm{SB}}+\epsilon L_{\mathrm{AGN}}}{\left(M_{\mathrm{H}_{2}} / \alpha\right)}
$$

where $L_{\mathrm{SB}}$ and $L_{\mathrm{AGN}}$ are the luminosities of the starburst and $\mathrm{AGN}$, respectively, and $\epsilon$ is the fraction of the AGN light absorbed by dust and reradiated in the thermal infrared. In the limit where either $L_{\mathrm{AGN}}=0$ or $\epsilon=0$, equation (5) is simply the star formation efficiency; i.e., if $\tau \gg 1$ toward the starburst population that is producing stars in steady state, $L_{\mathrm{IR}} / L_{\mathrm{CO}}^{\prime}$ is a measure of total energy output of the starburst population per unit of fuel available to form new stars. If $\epsilon=0$ for QSO hosts, then the high $L_{\mathrm{IR}} / L_{\mathrm{CO}}^{\prime}$ is an indication that they are producing stars extremely efficiently from the available molecular gas. Indeed, one would be forced to conclude that the star formation efficiency is preferentially higher in galaxies hosting luminous AGNs. The alternative interpretation is that $\epsilon>0$, and thus that $\epsilon L_{\mathrm{AGN}}$ contributes significantly to $L_{\mathrm{IR}}$.

\subsection{Dense Molecular Gas and Star Formation Rates}

The conclusions stated in the above discussion are only valid if $\mathrm{CO}$ is a robust tracer of star formation in these galaxies, i.e., if the $\mathrm{CO}$ emission is tracing most, if not all, of the molecular hydrogen that is actively forming stars. Given that $\mathrm{HCN}$ appears to be more closely coupled with star formation in starburst galaxies than $\mathrm{CO}$ (see $\S 1$ ), applying a similar analysis to $\mathrm{HCN}$ as done in $\S 5.1$ for CO will likely yield a more physically meaningful result.

Figure 5 is a plot of $L_{\mathrm{IR}} / L_{\mathrm{HCN}}^{\prime}$ versus $L_{\mathrm{IR}}$ of the QSO hosts, IRAS galaxies, and two high-redshift AGNs recently detected in $\mathrm{HCN}(1 \rightarrow 0)$. Again, the IRAS galaxies have been separated into those with warm and cool infrared colors. If the dust in the cool $I R A S$ galaxies is heated primarily by the starburst population

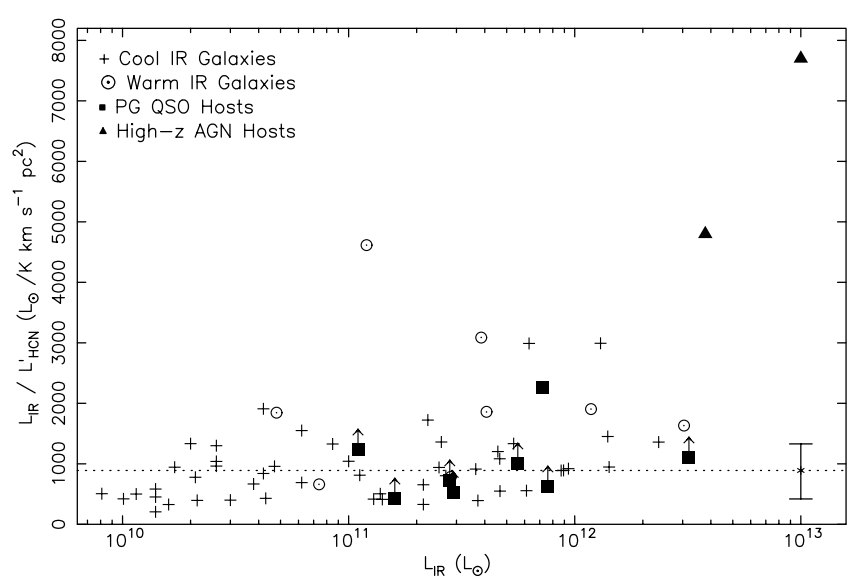

FIG. 5. $-L_{\mathrm{IR}} / L_{\mathrm{HCN}}^{\prime}$ vs. $L_{\mathrm{IR}}$ of the PG QSO hosts, a sample of local IRAS galaxies, and two high- $z$ AGNs (FSC 10214+4724 and the Cloverleaf Quasar). Squares with arrows represent $3 \sigma L_{\mathrm{IR}} / L_{\mathrm{HCN}}^{\prime}$ lower limits of the PG QSOs not detected in $\mathrm{HCN}$. The dashed line is $L_{\mathrm{IR}} / L_{\mathrm{HCN}}^{\prime}=890 L_{\odot}\left(\mathrm{K} \mathrm{km} \mathrm{s}^{-1} \mathrm{pc}^{2}\right)^{-1}$ (see $\S 5.1)$; the error bar associated with the dashed line encloses $67 \%(1 \sigma)$ of the cool galaxy data points. With the exception of NGC 4418 (Imanishi et al. 2004), Mrk 273 and IR 17208-0014 (Imanishi et al. 2006), and Arp 220 (this paper), the IRAS galaxy HCN data are compiled from Gao \& Solomon (2004a, 2004b). The F10214+4724 and Cloverleaf data are from Vanden Bout et al. (2004) and Solomon et al. (2003), respectively.

$(\epsilon \sim 0)$, then the median $L_{\mathrm{IR}} / L_{\mathrm{HCN}}^{\prime}$ of the cool IRAS sample is adopted as the ratio at which $L_{\mathrm{IR}}=L_{\mathrm{SB}}$; i.e.,

$$
\begin{aligned}
\left\langle\frac{L_{\mathrm{IR}}}{L_{\mathrm{HCN}}^{\prime}}\right\rangle_{\mathrm{cool}} & \sim \frac{L_{\mathrm{SB}}}{\left(M_{\mathrm{H}_{2}} / \alpha_{\mathrm{HCN}}\right)} \\
& \sim 890_{-470}^{+440} L_{\odot}\left(\mathrm{K} \mathrm{km} \mathrm{s}^{-1} \mathrm{pc}^{2}\right)^{-1}
\end{aligned}
$$

where $\alpha_{\mathrm{HCN}}$ is the $L_{\mathrm{HCN}}^{\prime}$-to- $M_{\mathrm{H}_{2}}$ mass conversion factor and the limits on $\left\langle L_{\mathrm{IR}} / L_{\mathrm{HCN}}^{\prime}\right\rangle_{\text {cool }}$ represent the range in which $67 \%$ (i.e., $1 \sigma$ ) of the data points around the median are contained.

The upper portion of Figure 5 (i.e., high $L_{\mathrm{IR}} / L_{\mathrm{HCN}}^{\prime}$ ) is mostly populated with the warm galaxies and AGN hosts. Specifically, the QSO host I Zw 1, the two high-redshift AGN hosts, and two of the eight warm IRAS galaxies have much higher $L_{\mathrm{IR}} / L_{\mathrm{HCN}}^{\prime}$ (>2200) than the cool galaxy population (i.e., low values of $L_{\mathrm{HCN}}^{\prime}$ for their $L_{\mathrm{IR}}$ ). These galaxies clearly have a significant contribution to their $L_{\mathrm{IR}}$ from AGN activity. Further, the fact that both of the cool IRAS galaxies within this $L_{\mathrm{IR}} / L_{\mathrm{HCN}}^{\prime}$ range (Arp 299 and Mrk 273, for which $L_{\mathrm{IR}} / L_{\mathrm{HCN}}^{\prime} \sim 3000$ ) have strong X-ray signatures of embedded AGNs (Della Ceca et al. 2002; Xia et al. 2002; Zesas et al. 2003) also supports the idea that high $L_{\mathrm{IR}} / L_{\mathrm{HCN}}^{\prime}$ ratios are the result of significant dust heating by AGNs. Four of the eight warm galaxies have $L_{\mathrm{IR}} / L_{\mathrm{HCN}}^{\prime}$ in the range 1600-2000, and thus overlap the high end of the bulk of the cool galaxy distribution. These galaxies, which include the well-studied ULIG Mrk 231, might have an equal mixture of AGN and starburst emission as viewed in the thermal infrared; however, they could also be almost entirely powered by star formation, as their $L_{\mathrm{IR}} / L_{\mathrm{HCN}}^{\prime}$ is not outside the range of that found in cool galaxies. Finally, two of the eight warm galaxies have $L_{\mathrm{IR}} / L_{\mathrm{HCN}}^{\prime}$ very near the median of the cool galaxy population, illustrating the fact that some fraction of the warm galaxy population have "normal" $L_{\mathrm{IR}} / L_{\mathrm{HCN}}^{\prime}$. Whether or not the PG QSO hosts not detected in HCN have high $L_{\mathrm{IR}} / L_{\mathrm{HCN}}^{\prime}$ similar to I Zw 1 cannot be addressed with the data in Figure 5.

While only one of the eight PG QSO hosts was detected in $\mathrm{HCN}$, and the $L_{\mathrm{IR}} / L_{\mathrm{HCN}}^{\prime}$ lower limits of the undetected QSO hosts are not sufficiently constraining to be conclusive, the $L_{\mathrm{IR}} / L_{\mathrm{HCN}}^{\prime}$ ratios 


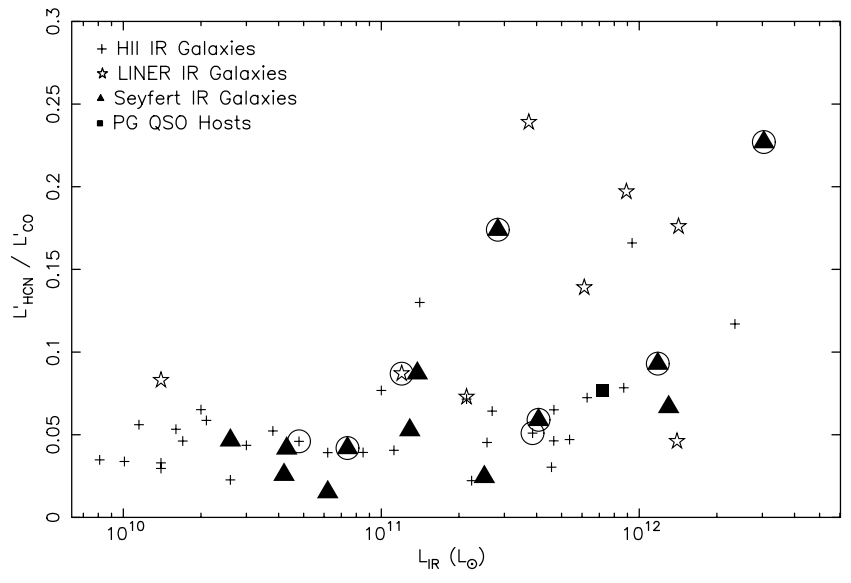

FIG. 6. $-L_{\mathrm{HCN}}^{\prime} / L_{\mathrm{CO}}^{\prime}$ vs. $L_{\mathrm{IR}}$ for I Zw 1 and IRAS galaxies. The IRAS galaxy data are plotted by their optical emission-line classification. The warm IRAS galaxies are circled.

of the undetected hosts can possibly be inferred by making use of the larger number of $\mathrm{HCN}$-detected Seyfert galaxies in the IRAS galaxy sample. Figure 6 is a plot of $L_{\mathrm{HCN}}^{\prime} / L_{\mathrm{CO}}^{\prime}$ versus $L_{\mathrm{IR}}$, and is a modified version of Figure 4 in Gao \& Solomon (2004b). Here, the IRAS galaxies have been plotted in terms of their optical emission-line classifications (i.e., Seyfert, LINER [low-ionization nuclear emission region], and $\mathrm{H}$ II region-like galaxies). In this figure there is an increased spread in $L_{\mathrm{HCN}}^{\prime} / L_{\mathrm{CO}}^{\prime}$ values for galaxies with $L_{\mathrm{IR}}>10^{11} L_{\odot}$, which Gao \& Solomon (2004b) attribute to the increase in the fraction of dense gas in luminous infrared galaxies corresponding to the increase in starburst activity. Eleven of the 13 (85\%) Seyfert galaxies in Figure 6 have $L_{\mathrm{HCN}}^{\prime} / L_{\mathrm{CO}}^{\prime}<0.1$. In addition, I Zw 1 has an $L_{\mathrm{HCN}}^{\prime} / L_{\mathrm{CO}}^{\prime}$ ratio within the distribution of Seyfert galaxy data points at $L_{\mathrm{HCN}}^{\prime} / L_{\mathrm{CO}}^{\prime}<0.1$.
Given this, it is a reasonable assumption that the PG QSO hosts in the present sample have low $L_{\mathrm{HCN}}^{\prime} / L_{\mathrm{CO}}^{\prime}$ similar to the bulk of the Seyfert galaxies in Figure 6. Thus, for the PG QSO hosts, the median $L_{\mathrm{HCN}}^{\prime} / L_{\mathrm{CO}}^{\prime}$ of Seyfert galaxies and I Zw 1 in Figure 6, i.e.,

$$
\left\langle\frac{L_{\mathrm{HCN}}^{\prime}}{L_{\mathrm{CO}}^{\prime}}\right\rangle_{\text {Seyfert }} \sim 0.06_{-0.04}^{+0.03}
$$

is adopted, where the uncertainties span the range of $L_{\mathrm{HCN}}^{\prime} / L_{\mathrm{CO}}^{\prime}$ for the 11 low $L_{\mathrm{HCN}} / L_{\mathrm{CO}}^{\prime}$ Seyfert galaxies. A direct estimation of $L_{\mathrm{IR}} / L_{\mathrm{HCN}}^{\prime}$ for the QSO hosts can then be made from the $L_{\mathrm{IR}} / L_{\mathrm{CO}}^{\prime}$ of the QSO hosts listed in Table 3 . The resulting $L_{\mathrm{IR}} / L_{\mathrm{HCN}}^{\prime}$ ratios are listed in Table 4, and the data are plotted in Figure 7. The stellar contributions to the production of $L_{\mathrm{IR}}$ of PG QSOs hosts is thus

$$
\begin{aligned}
\Delta_{\mathrm{SB}}(\mathrm{IR}) & \sim 5340\left(\frac{\left\langle L_{\mathrm{IR}} / L_{\mathrm{HCN}}^{\prime}\right\rangle_{\text {cool }}}{890}\right) \\
& \times\left(\frac{\left\langle L_{\mathrm{HCN}}^{\prime} / L_{\mathrm{CO}}^{\prime}\right\rangle_{\text {Seyfert }}}{0.06}\right) \\
& \times\left(\frac{L_{\odot}\left[\mathrm{K} \mathrm{km} \mathrm{s}^{-1} \mathrm{pc}^{2}\right]^{-1}}{L_{\mathrm{IR}} / L_{\mathrm{CO}}^{\prime}}\right) \% \\
& \sim 7 \%-39 \%,
\end{aligned}
$$

and the star formation rates are $2-37 M_{\odot} \mathrm{yr}^{-1}$ (median $=10 M_{\odot}$ $\mathrm{yr}^{-1}$ ). Note, however, that given the uncertainties in equation (7), the possibility that massive, young stars produce $60 \%$ of $L_{\mathrm{IR}}$ cannot be ruled out for $50 \%$ of the QSOs plotted in Figure 7. By comparison, the corresponding values for the warm galaxies are

\begin{tabular}{|c|c|c|c|c|c|c|c|}
\hline Source & Type & Class $^{\mathrm{a}}$ & $L_{\mathrm{IR}} / L_{\mathrm{HCN}}^{\prime}{ }^{\mathrm{b}}$ & $L_{\mathrm{FIR}} / L_{\mathrm{HCN}}^{\prime} \mathrm{b}$ & $\left\langle L_{\mathrm{IR}} / L_{\mathrm{HCN}}^{\prime}\right\rangle_{\text {cool }} /\left(L_{\mathrm{IR}} / L_{\mathrm{HCN}}^{\prime}\right)$ & $\left\langle L_{\mathrm{FIR}} / L_{\mathrm{HCN}}^{\prime}\right\rangle_{\mathrm{cool}} /\left(L_{\mathrm{FIR}} / L_{\mathrm{HCN}}^{\prime}\right)$ & $\mathrm{SFR}^{\mathrm{c}}$ \\
\hline 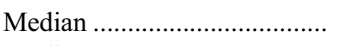 & Cool & $\cdots$ & $890_{-470}^{+440}$ & $660_{-330}^{+370}$ & & & \\
\hline 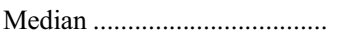 & Warm & $\ldots$ & $1850_{-1070}^{+1240}$ & $1220_{-810}^{+860}$ & $0.48 \pm 0.25$ & $0.54 \pm 0.28$ & $\ldots$ \\
\hline 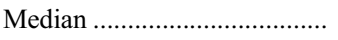 & QSO & $\ldots$ & $3150_{-1050}^{+6300}$ & $1575_{-530}^{+4720}$ & $0.28_{-0.24}^{+0.35}$ & $0.42_{-0.35}^{+0.56}$ & \\
\hline 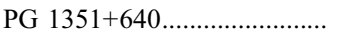 & QSO & Seyfert 1 & $12120_{-4040}^{+2430}$ & $4540_{-1520}^{+9090}$ & $0.07_{-0.06}^{+0.09 \mathrm{~d}}$ & $0.15_{-0.12}^{+0.19 \mathrm{~d}}$ & $5_{-5}^{+7}$ \\
\hline PG $0157+001 \ldots \ldots \ldots \ldots \ldots \ldots$ & QSO & Seyfert 1 & $10120_{-3370}^{+2030}$ & $6640_{-2210}^{+13280}$ & $0.09_{-0.07}^{+0.11 \mathrm{~d}}$ & $0.10_{-0.08}^{+0.13 \mathrm{~d}}$ & $37_{-31}^{+46}$ \\
\hline 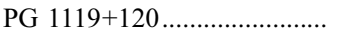 & QSO & Seyfert 1 & $7530_{-2510}^{+15070}$ & $3010_{-1000}^{+6030}$ & $0.12_{-0.10}^{+0.15 \mathrm{~d}}$ & $0.22_{-0.18}^{+0.29 \mathrm{~d}}$ & $2_{-1}^{+2}$ \\
\hline Cloverleaf Quasar ....................... & High- $z$ AGN & Seyfert 1 & 7700 & 1700 & $0.12 \pm 0.06$ & $0.39 \pm 0.20$ & $375 \pm 190$ \\
\hline PG 2130+099 ............................ & QSO & Seyfert 1 & $6250_{-2080}^{+12500}$ & $1840_{-610}^{+3690}$ & $0.14_{-0.12}^{+0.18 \mathrm{~d}}$ & $0.36_{-0.30}^{+0.48 \mathrm{~d}}$ & $4_{-3}^{+5}$ \\
\hline IRAS FSC $10214+4724 \ldots \ldots$. & High- $z$ AGN & Seyfert 2 & 4800 & 2600 & $0.19 \pm 0.10$ & $0.25 \pm 0.13$ & $223 \pm 110$ \\
\hline 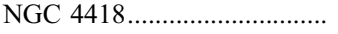 & Warm & LINER & 4610 & 3830 & $0.19 \pm 0.10$ & $0.17 \pm 0.09$ & $3 \pm 2$ \\
\hline PG $1440+356 \ldots \ldots \ldots \ldots \ldots \ldots \ldots$ & QSO & Seyfert 1 & $3150_{-1050}^{+6300}$ & $1580_{-520}^{+3150}$ & $0.28_{-0.24}^{+0.35 \mathrm{~d}}$ & $0.42_{-0.35}^{+0.56 \mathrm{~d}}$ & $10_{-9}^{+13}$ \\
\hline 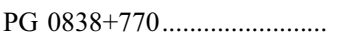 & QSO & Seyfert 1 & $3150_{-1050}^{+6300}$ & $1520_{-510}^{+3040}$ & $0.28_{-0.24}^{+0.35 \mathrm{~d}}$ & $0.43_{-0.36}^{+0.58 \mathrm{~d}}$ & $11_{-9}^{+13}$ \\
\hline 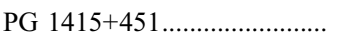 & QSO & Seyfert 1 & $2720_{-910}^{+5430}$ & $1120_{-370}^{+2240}$ & $0.33_{-0.28}^{+0.41 d}$ & $0.59_{-0.49}^{+0.79 \mathrm{~d}}$ & $7_{-6}^{+9}$ \\
\hline PG $1613+658 \ldots \ldots \ldots \ldots \ldots$ & QSO & Seyfert 1 & $2630_{-880}^{+5270}$ & $1380_{-460}^{+2760}$ & $0.34_{-0.28}^{+0.42 \mathrm{~d}}$ & $0.48_{-0.40}^{+0.64 \mathrm{~d}}$ & $34_{-29}^{+42}$ \\
\hline 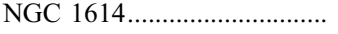 & Warm & H II/LINER & 3090 & 2090 & $0.29 \pm 0.15$ & $0.32 \pm 0.17$ & $15 \pm 7$ \\
\hline PG $0050+124=\mathrm{I} Z \mathrm{Zw} 1 \ldots \ldots$ & QSO & Seyfert 1 & 2260 & 880 & $0.39 \pm 0.20$ & $0.75 \pm 0.39$ & $37 \pm 19$ \\
\hline IRAS FSC $05189-2524 \ldots \ldots$. & Warm & Seyfert 2 & 1900 & 1260 & $0.47 \pm 0.24$ & $0.52 \pm 0.28$ & $73 \pm 37$ \\
\hline 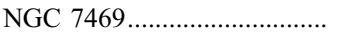 & Warm & Seyfert 1 & 1860 & 1190 & $0.48 \pm 0.25$ & $0.55 \pm 0.29$ & $26 \pm 13$ \\
\hline NGC $3034=$ M82 .................. & Warm & H II & 1850 & 1270 & $0.48 \pm 0.25$ & $0.52 \pm 0.27$ & $3 \pm 2$ \\
\hline Mrk 231 & Warm & Seyfert 1 & 1630 & 1030 & $0.55 \pm 0.28$ & $0.64 \pm 0.34$ & $219 \pm 112$ \\
\hline 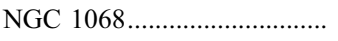 & Warm & Seyfert 2 & 780 & 320 & $1.1 \pm 0.59$ & $2.0 \pm 1.1$ & $42 \pm 21$ \\
\hline NGC $7479 \ldots \ldots \ldots \ldots \ldots \ldots$ & Warm & Seyfert 2 & 660 & 410 & $1.4 \pm 0.70$ & $1.6 \pm 0.83$ & $13 \pm 7$ \\
\hline
\end{tabular}
$\sim 10 \%-100 \%$ and $\sim 3-220 M_{\odot} \mathrm{yr}^{-1}$ (median $\left.=20 M_{\odot} \mathrm{yr}^{-1}\right)$, respectively.

TABLE 4

Infrared-to-HCN Luminosity Ratio Comparisons and Adjusted Star Formation Rates

\footnotetext{
${ }^{a}$ References for optical spectral classification: Veilleux et al. (1995) and Ho et al. (1997).

${ }^{\mathrm{b}}$ In units of $L_{\odot}\left(\mathrm{K} \mathrm{km} \mathrm{s}^{-1} \mathrm{pc}^{2}\right)^{-1}$.

${ }^{c}$ Star formation rate in $M_{\odot} \mathrm{yr}^{-1}$.

d Calculated by adopting $L_{\mathrm{HCN}}^{\prime} / L_{\mathrm{CO}}^{\prime}=0.06$ (see $\left.\S 5.1\right)$.
} 


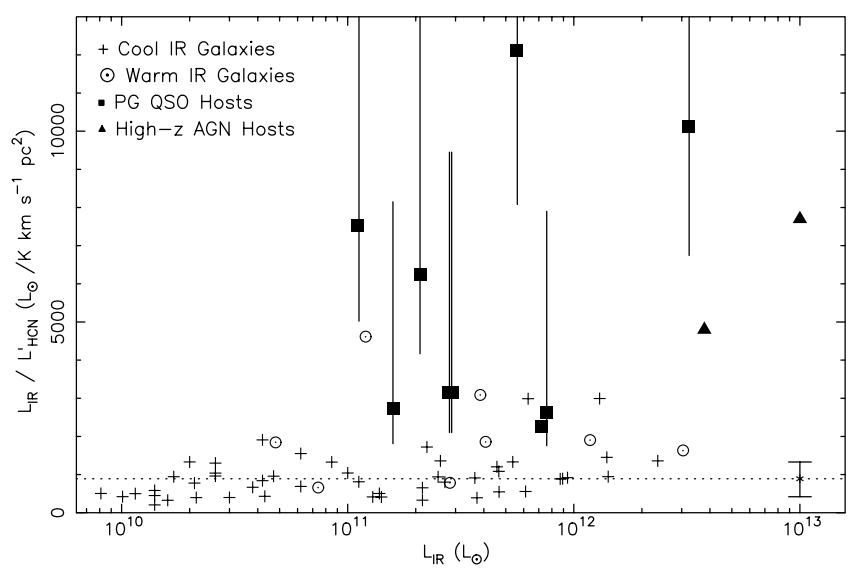

FIG. 7.- Same as Fig. 5, except the estimated $L_{\mathrm{IR}} / L_{\mathrm{HCN}}^{\prime}(\mathrm{see} \S 5.2)$ of the PG QSO hosts not detected in HCN are plotted and the $y$-axis upper limit has been increased. The vertical bars associated with the PG QSO host data points cover the range in $L_{\mathrm{IR}} / L_{\mathrm{HCN}}^{\prime}$ that results from the uncertainties in eq. (7).

Clearly, a significant fraction of the cool IRAS galaxies do harbor AGNs, which may or may not contribute significantly to the heating of dust in their host galaxy. Thus, as a double-check of the results derived from Figure 7, the figure has been replotted with the cool IRAS galaxies optically classified as Seyferts and LINER galaxies ${ }^{5}$ omitted (Fig. 8). Galaxies with transitional spectra (e.g., galaxies classified both as LINER galaxies and $\mathrm{H}$ II region-like galaxies via different line diagnostics) have been retained, and galaxies with no published classification have also been omitted. Finally, Arp 299, which has an $\mathrm{H}$ II region-like optical classification but is known via X-ray observations to harbor an $\mathrm{AGN}$, has been omitted. The main outcome of plotting only the remaining $\mathrm{H}$ II region-like galaxies and transitional galaxies is the removal of the outliers in the $L_{\mathrm{IR}} / L_{\mathrm{HCN}}^{\prime}$ distribution. The value of $\left\langle L_{\mathrm{IR}} / L_{\mathrm{HCN}}^{\prime}\right\rangle_{\text {cool }}(=910 \pm 430)$ remains essentially unchanged.

\subsection{Dense Molecular Gas and $L_{\mathrm{FIR}}$}

Another obvious issue to consider is whether the above results differ if the far-infrared emission from cool galaxies is considered

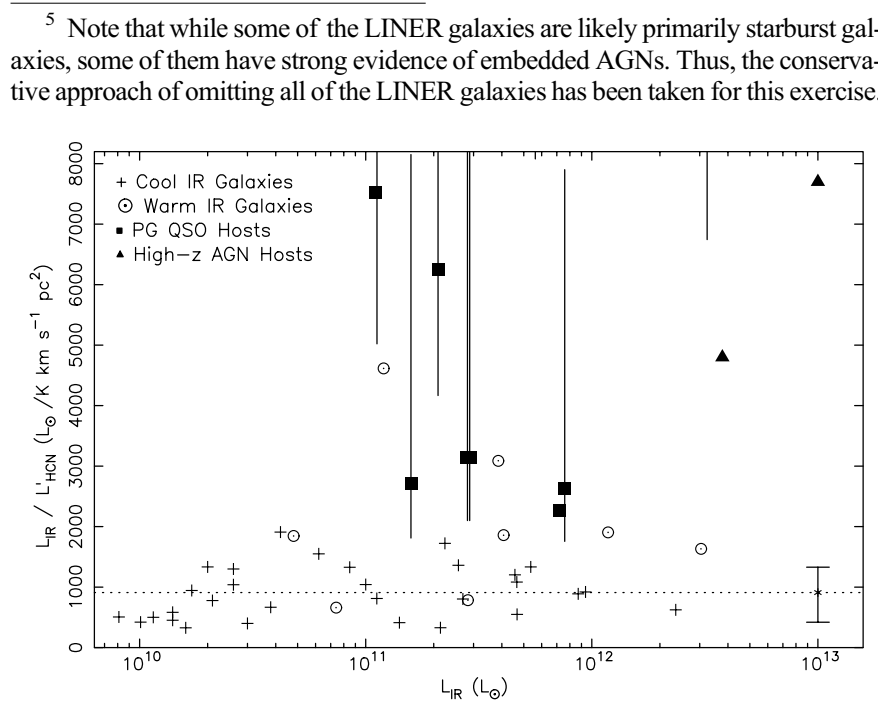

FIG. 8.- Same as Fig. 7, except only the cool IRAS galaxies optically classified as H II region-like galaxies or transition galaxies are plotted (Arp 299 has also been omitted; see text). The warm galaxies and PG QSO host data points remain unchanged. Optical emission-line classifications are obtained from Veilleux et al. (1995), Ho et al. (1997), Mazzarella \& Boroson (1993), and the NASA/ IPAC Extragalactic Database.

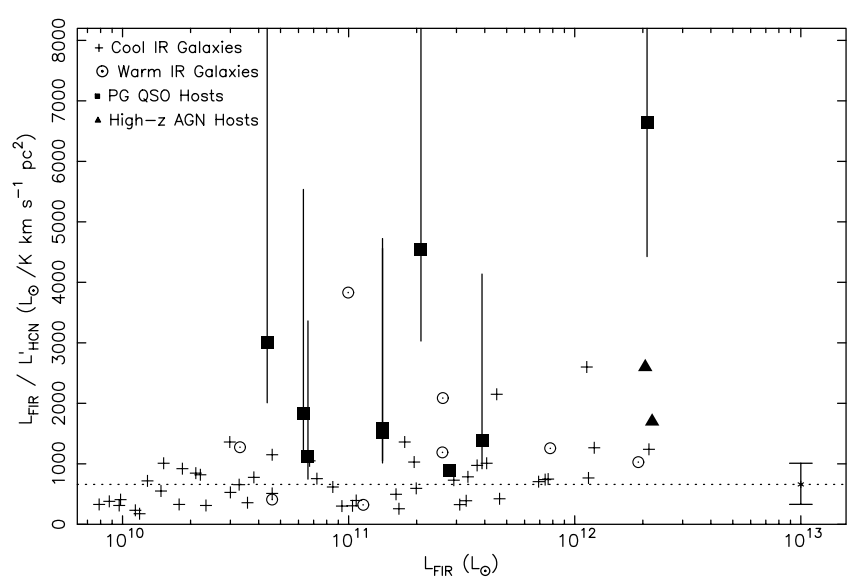

FIG. 9. $-L_{\mathrm{FIR}} / L_{\mathrm{HCN}}^{\prime}$ vs. $L_{\mathrm{FIR}}$ of the PG QSO hosts, a sample of local IRAS galaxies, and two high- $z$ AGNs (FSC 10214+4724 and the Cloverleaf Quasar). The dashed line is $L_{\mathrm{FIR}} / L_{\mathrm{HCN}}^{\prime}=660 L_{\odot}\left(\mathrm{K} \mathrm{km} \mathrm{s}^{-1} \mathrm{pc}^{2}\right)^{-1}$ (see $\left.\S 5.3\right)$; the error bar associated with the dashed line encloses $67 \%(1 \sigma)$ of the cool galaxy data points.

as the star formation component of the total infrared emission, i.e., if $\left\langle L_{\mathrm{FIR}} / L_{\mathrm{HCN}}^{\prime}\right\rangle_{\text {cool }} \sim L_{\mathrm{SB}} / L_{\mathrm{HCN}}^{\prime}$. Figure 9 is a plot of $L_{\mathrm{FIR}} / L_{\mathrm{HCN}}^{\prime}$ versus $L_{\mathrm{FIR}}$ of the same samples plotted in Figure 7 . The use of $L_{\mathrm{FIR}}$ instead of $L_{\mathrm{IR}}$ reduces the disparity between the PG QSO and high- $z$ AGN host data points relative to the IRAS galaxy data points and shows the possibility that the far-infrared emission in several PG QSO hosts could be due almost entirely to dust heated by young, massive stars (see Table 4). In particular, I Zw 1 has a $L_{\mathrm{FIR}} / L_{\mathrm{HCN}}^{\prime}$ very near the median value of the cool IRAS galaxy population. In the case of the warm galaxies, the median $\left\langle L_{\mathrm{FIR}} / L_{\mathrm{HCN}}^{\prime}\right\rangle_{\text {warm }}\left(\sim 1220_{-810}^{+860}\right)$ is $33 \%$ lower than $\left\langle L_{\mathrm{IR}} / L_{\mathrm{HCN}}^{\prime}\right\rangle_{\text {warm }}$; however, the median value of the cool IRAS galaxies, $\left\langle L_{\mathrm{FIR}} / L_{\mathrm{HCN}}^{\prime}\right\rangle_{\text {cool }} \sim 660_{-330}^{+370}$, is $25 \%$ lower than $\left\langle L_{\mathrm{IR}} / L_{\mathrm{HCN}}^{\prime}\right\rangle_{\text {cool }}$. Thus, the relative spacing of the warm and cool galaxy data points is only marginally changed by using $L_{\mathrm{FIR}}$. As a result, the contribution of dust heating by young, massive stars to the production of $L_{\mathrm{FIR}}$ for the PG QSO hosts and warm galaxies increases by $1 \%-35 \%$ and $1 \%-12 \%$, respectively, over the contribution derived for $L_{\mathrm{IR}}$ (see Table 4). These results reflect the fact that the average $L_{\mathrm{FIR}} / L_{\mathrm{IR}}$ for the cool, warm, and PG QSO galaxies in Figure 8 are $0.77 \pm 0.05,0.64 \pm 0.11$, and $0.44 \pm 0.10$, respectively.

\subsection{Comparison with Recent Estimates of Star Formation Rates of PG QSO Hosts}

The general conclusion that the PG QSO host star formation rates in equation (4) may, in some cases, be overestimates is consistent with a recent study of the star formation rates of QSO hosts by Ho (2005). In this study, star formation rates were derived by first assuming that the $[\mathrm{O}$ II] $23727 /[\mathrm{O}$ III] 25007 line ratio in QSOs is the same as the constant value measured for the narrowline regions of Seyfert galaxies, then by attributing excesses in the ratio to star formation within the host. Doing so yields [O II] 23727-derived star formation rates of $\sim 0.6-20 M_{\odot} \mathrm{yr}^{-1}$; i.e., significantly lower than what would be derived based solely on the assumption that the $L_{\mathrm{IR}} \sim L_{\mathrm{SB}}$ and consistent with the low end of the range in star formation rates estimated for individual hosts in Table 4. Note, however, that the [O II] 23727 emission line is subject to significant extinction by dust, and thus the Ho (2005) estimates are likely lower limits.

Another study (Schweitzer et al. 2006) addresses the issue of the nature of far-infrared emission in PG QSO hosts by applying the $7.7 \mu \mathrm{m}$ polycyclic aromatic hydrocarbon $(\mathrm{PAH})$-to-far-infrared luminosity ratio $\left(L_{\mathrm{PAH}} / L_{\mathrm{FIR}}\right)$ versus [Ne II] $12.5 \mu \mathrm{m}$-to-far-infrared 
luminosity ratio $\left(L_{[\mathrm{Ne} \mathrm{II}} / L_{\mathrm{FIR}}\right)$ diagnostic of starburst galaxies to the QSO host sample. Starburst galaxies are observed to have strong PAH and weak [ $\mathrm{Ne}$ II] relative to AGN-dominated hosts because the AGN efficiently destroys PAHs and is able to produce extended semi-ionized regions in which neon is collisionally excited. Schweitzer et al. concluded that $30 \%$ or more of the far-infrared emission is due to dust heating by young, massive stars. By comparison, a median percentage of $42_{-35}^{+56 \%}$ is estimated from the present analysis of the HCN data.

\subsection{The Effects of the AGN on $\mathrm{HCN}$ Emission}

Throughout this paper the assumption has been made that $\mathrm{HCN}$ is collisionally excited by molecular hydrogen. Recent papers have challenged this claim by stating that in galaxies harboring AGNs, X-ray emission from the AGN enhances HCN emission (e.g., Kohno et al. 2003; Gracia-Carpio et al. 2006). The high $L_{\mathrm{IR}} / L_{\mathrm{HCN}}^{\prime}$, and thus the low $L_{\mathrm{HCN}}^{\prime}$, of most of the PG QSO hosts, high- $z$ AGN hosts, and warm galaxies relative to cool IRAS galaxies (Fig. 7) clearly contradict this hypothesis.

To examine this issue another way, consider again the plot of $L_{\mathrm{HCN}}^{\prime} / L_{\mathrm{CO}}^{\prime}$ versus $L_{\mathrm{IR}}$ of the QSO hosts and IRAS galaxies (Fig. 6). In this figure I Zw 1 and most of the Seyfert galaxies have $L_{\mathrm{HCN}}^{\prime} / L_{\mathrm{CO}}^{\prime}<0.1$, consistent with the distribution of the $\mathrm{H}$ II region-like galaxies. Two of the Seyfert galaxies have $L_{\mathrm{HCN}}^{\prime} / L_{\mathrm{CO}}^{\prime}>$ 0.1 ; however, they are still within the distribution of $L_{\mathrm{HCN}}^{\prime} / L_{\mathrm{CO}}^{\prime}$ of $\mathrm{H}$ II region-like and LINER galaxies. Thus, there is no evidence that the global $\mathrm{HCN}$ emission is enhanced relative to $\mathrm{CO}$ by the presence of a powerful AGN.

\section{CONCLUSIONS}

New CO and HCN observations of a sample of $z<0.17$ optically selected PG QSO hosts with infrared excesses are presented. These data have been compared with similar data of $I R A S$-detected galaxies to assess the validity of using the infrared luminosity to estimate star formation rates in luminous AGN hosts. The following conclusions are reached:

1. The new CO measurements confirm the results presented in Evans et al. (2001), that QSO hosts as a class may have low values of $L_{\mathrm{CO}}^{\prime}$ for their $L_{\mathrm{IR}}$ relative to IRAS galaxies, or relatively high values of $L_{\mathrm{IR}} / L_{\mathrm{CO}}^{\prime}$. Such high $L_{\mathrm{IR}} / L_{\mathrm{CO}}^{\prime}$ ratios may be evidence that AGNs contribute significantly to the heating of dust in their host galaxies, or that they have high star formation efficiencies.

2. A comparison of the QSO hosts and IRAS galaxies surveyed in $\mathrm{HCN}(1 \rightarrow 0)$ to date show the upper distribution of $L_{\mathrm{IR}} / L_{\mathrm{HCN}}^{\prime}(>1600)$ to be mostly populated by warm IRAS galaxies and AGN hosts. This distribution overlaps at the low end with cool IRAS galaxies for which $\left\langle L_{\mathrm{IR}} / L_{\mathrm{HCN}}^{\prime}\right\rangle_{\text {cool }} \sim 890_{-470}^{+440} L_{\odot}$ $\left(\mathrm{K} \mathrm{km} \mathrm{s}^{-1} \mathrm{pc}^{2}\right)^{-1}$. There are also two (out of eight) warm galaxies with $L_{\mathrm{IR}} / L_{\mathrm{HCN}}^{\prime}$ comparable to the median $L_{\mathrm{IR}} / L_{\mathrm{HCN}}^{\prime}$ of cool galaxies. An enhanced $L_{\mathrm{IR}} / L_{\mathrm{HCN}}^{\prime}$ relative to cool IRAS galaxies thus appears to be an indication that an AGN is contributing significantly to heating the dust. However, the possibility that the dust in most of the QSO hosts and warm galaxies is heated primarily by young, massive stars cannot be entirely ruled out. If the median ratio of $L_{\mathrm{HCN}}^{\prime} / L_{\mathrm{CO}}^{\prime} \sim 0.06$ observed for Seyfert galaxies and the PG QSO host I Zw 1 is applied to the PG QSOs not detected in $\mathrm{HCN}$, then the derived $L_{\mathrm{IR}} / L_{\mathrm{HCN}}^{\prime}$ ratios correspond to a stellar contribution to the production of $L_{\mathrm{IR}}$ of $\sim 7 \%-35 \%$ and star formation rates of $\sim 2-37 M_{\odot} \mathrm{yr}^{-1}$ are derived for the QSO hosts. The corresponding values for the warm galaxies are $\sim 10 \%-100 \%$ and $\sim 3-220 M_{\odot} \mathrm{yr}^{-1}$.

3. The average $\left\langle L_{\mathrm{FIR}} / L_{\mathrm{IR}}\right\rangle$ of the cool, warm, and PG QSO galaxies considered in this study is $0.77 \pm 0.05,0.64 \pm 0.11$, and $0.44 \pm 0.10$, respectively. Thus, the disparity between the QSO (and high-redshift AGN) hosts and the cool galaxies is diminished by comparing the $L_{\mathrm{FIR}}$ of QSO hosts and cool galaxies. If the far-infrared is adopted as the star formation component of the total infrared in cool galaxies, i.e., $\left\langle L_{\mathrm{FIR}} / L_{\mathrm{HCN}}^{\prime}\right\rangle_{\mathrm{cool}} \sim$ $L_{\mathrm{SB}} / L_{\mathrm{HCN}}^{\prime}$, then five of the nine QSO hosts, including I Zw 1 , potentially have all of their $L_{\mathrm{FIR}}$ generated by dust heated by massive stars. The stellar contributions to $L_{\mathrm{FIR}}$ in QSO hosts are up to $35 \%$ higher than those derived for $L_{\mathrm{IR}}$, but the warm galaxies have contributions only $10 \%$ higher than those derived for $L_{\mathrm{IR}}$.

4. The PG QSO host I Zw 1 and the IRAS galaxies with Seyfert optical classifications have $L_{\mathrm{HCN}}^{\prime} / L_{\mathrm{CO}}^{\prime}$ distributions comparable to $\mathrm{H}$ in region-like galaxies. It is thus unlikely that the global $\mathrm{HCN}$ emission in galaxies harboring luminous AGNs is enhanced relative to $\mathrm{CO}$ by radiation from the $\mathrm{AGN}$.

An HCN survey of a more statistically complete sample of IRAS galaxies and PG QSO hosts is required to assess the robustness of many of these results. The apparent faintness of $\mathrm{HCN}$ emission in PG QSO hosts, and in many warm galaxies, necessitates the use of a larger millimeter-wave telescope. Such observations may be possible with the soon-to-be commissioned $50 \mathrm{~m}$ diameter Large Millimeter Telescope or the $100 \mathrm{~m}$ diameter Green Bank Telescope (GBT). Frequencies as high as $90 \mathrm{GHz}$ should be accessible with the GBT in a few years.

We thank the telescope operators and staff of the IRAM $30 \mathrm{~m}$ telescope for their support both during and after the observations and the anonymous referee for critical comments that greatly improved the accuracy and clarity of the paper. A. S. E. also thanks J. Mazzarella, T. Bertram, and J. Mulchaey for useful discussions and assistance. A. S. E. was supported by NSF grant AST 02-06262. This research has made use of the NASA/IPAC Extragalactic Database, which is operated by the Jet Propulsion Laboratory, California Institute of Technology, under contract with the National Aeronautics and Space Administration.

\section{REFERENCES}

Alloin, D., Barvainis, R., Gordon, M. A., \& Antonucci, R. R. J. 1992, A\&A, 265,429

Barvainis, R., Alloin, D., \& Antonucci, R. 1989, ApJ, 337, L69

Della Ceca, R., et al. 2002, ApJ, 581, L9

Elitzur, M., Nenkova, M., \& Ivezic, Z. 2004, in ASP Conf. Ser. 320, The Neutral ISM in Starburst Galaxies, ed. S. Aalto, S. Huettemeister, \& A. Pedlar (San Francisco: ASP), 242

Evans, A. S. 2003, in ASP Conf. Ser. 320, The Neutral ISM in Starburst Galaxies, ed. S. Aalto, S. Huettemeister, \& A. Pedlar (San Francisco: ASP), 222

2006a, in Extreme Starbursts, Near and Far, ed. Y. Gao \& D. Sanders

(Publ. Purple Mountain Obs.), in press

. 2006b, NewA Rev., in press

Evans, A. S., Frayer, D. T., Surace, J. A., \& Sanders, D. B. 2001, AJ, 121, 3285

Evans, A. S., Mazzarella, J. M., Surace, J. A., Frayer, D. T., Iwasawa, I., \& Sanders, D. B. 2005, ApJS, 159, 197

Gao, Y., \& Solomon, P. M. 2004a, ApJS, 152, 63 2004b, ApJ, 606, 271

Gracia-Carpio, J., Garcia-Burillo, S., Planesas, P., \& Colina, L. 2006, ApJ, 640, L135

Guyon, O., Sanders, D. B., \& Stockton, A. 2006, ApJS, 166, 89

Haas, M., et al. 2003, A\&A, 402, 87

Ho, L. 2005, ApJ, 629, 680

Ho, L., Filippenko, A. V., \& Sargent, W. L. 1997, ApJS, 112, 315

Imanishi, M., Nakanishi, K., \& Kohno, K. 2006, AJ, 131, 2888

Imanishi, M., Nakanishi, K., Kuno, N., \& Kohno, K. 2004, AJ, 128, 2037 
Kennicutt, R. C., Jr. 1998, ARA\&A, 36, 189

Kohno, K., Ishizuki, S., Matsushita, S., Vila-Vilaro, B., \& Kawabe, R. 2003, PASJ, 55, L1

Mazzarella, J. M., \& Boroson, T. A. 1993, ApJS, 85, 27

Mazzarella, J. M., Graham, J. R., Sanders, D. B., \& Djorgovski, S. 1993, ApJ, 409,170

Sanders, D. B., Mazzarella, J. M., Kim, D. C., Surace, J. A., \& Soifer, B. T. 2003, AJ, 126, 1607

Sanders, D. B., \& Mirabel, I. F. 1996, ARA\&A, 34, 749

Sanders, D. B., Phinney, E. S., Neugebauer, G., Soifer, B. T., \& Matthews, K. 1989, ApJ, 347, 29

Sanders, D. B., Scoville, N. Z., \& Soifer, B. T. 1988a, ApJ, 335, L1

Sanders, D. B., Soifer, B. T., Elias, J. H., Madore, B. F., Matthews, K., Neugebauer, G., \& Scoville, N. Z. 1988b, ApJ, 325, 74

Sanders, D. B., Soifer, B. T., Elias, J. H., Neugebauer, G., \& Matthews, K. 1988c, ApJ, 328, L35

Schmidt, M., \& Green, R. F. 1983, ApJ, 269, 352
Schweitzer, M., et al. 2006, ApJ, 649, 79

Scoville, N. Z., Frayer, D. T., Schinnerer, E., \& Christopher, M. 2003, ApJ, $585, \mathrm{~L} 105$

Solomon, P. M., Downes, D., Radford, S. J. E., \& Barrett, J. W. 1997, ApJ, 478, 144

Solomon, P. M., Vanden Bout, P. A., Carilli, C., \& Guelin, M. 2003, Nature, 426, 636

Stockton, A., \& MacKenty, J. W. 1983, Nature, 305, 678

Surace, J. A., Sanders, D. B., \& Evans, A. S. 2001, AJ, 122, 2791

Vanden Bout, P. A., Solomon, P. M., \& Maddalena, R. J. 2004, ApJ, 614, L97

Veilleux, S., Kim, D.-C., Sanders, D. B., Mazzarella, J. M., \& Soifer, B. T. 1995, ApJS, 98, 171

Veilleux, S., et al. 2006, ApJ, 643, 707

Xia, X. Y., Xue, S. J., Mao, S., Boller, Th., Deng, Z. G., \& Wu, H. 2002, ApJ, 564, 196

Zezas, A., Ward, M. J., \& Murray, S. S. 2003, ApJ, 594, L31 